\title{
TOUGH: Observational aspects of gamma-ray burst host galaxies
}

\author{
Jens Hjorth ${ }^{* a}$ Daniele Malesani, ${ }^{a}$ Andreas O. Jaunsen, ${ }^{b}$ Andrew J. Levan, ${ }^{c}$ \\ Bo Milvang-Jensen, ${ }^{a}$ Darach Watson, ${ }^{a}$ Javier Gorosabel, ${ }^{d}$ Johan P. U. Fynbo, ${ }^{a}$ \\ Michał J. Michałowski, ${ }^{e}$ Nial R. Tanvir, ${ }^{f}$ Páll Jakobsson, ${ }^{g}$ Palle Møller, ${ }^{h}$ \\ Steve Schulze ${ }^{g}$ and Thomas Krühler ${ }^{a}$ \\ a Dark Cosmology Centre, Niels Bohr Institute, University of Copenhagen \\ DK-2100 Copenhagen, Denmark \\ ${ }^{b}$ Institute of Theoretical Astrophysics, University of Oslo \\ PO Box 1029 Blindern, N-0315 Oslo, Norway \\ ${ }^{c}$ Department of Physics, University of Warwick \\ Coventry CV4 7AL, UK \\ ${ }^{d}$ Instituto de Astrofísica de Andalucía (IAA-CSIC) \\ P.O. Box 03004, E-18080 Granada, Spain \\ e SUPA, Institute for Astronomy, University of Edinburgh, Royal Observatory \\ Edinburgh, EH9 3HJ, UK \\ ${ }^{f}$ Department of Physics and Astronomy, University of Leicester \\ University Road, Leicester LE1 7RH, UK \\ ${ }^{g}$ Centre for Astrophysics and Cosmology, Science Institute, University of Iceland \\ Dunhagi 3, 107 Reykjavík, Iceland \\ ${ }^{h}$ European Southern Observatory \\ Karl-Schwarzschild-Str. 2, D-85748 Garching by München, Germany \\ E-mail: jens@dark-cosmology.dk, malesani@dark-cosmology.dk, \\ ajaunsen@gmail.com, A.J.Levan@warwick.ac.uk, \\ milvang@dark-cosmology.dk, darach@dark-cosmology.dk, jgu@iaa.es, \\ jfynbo@dark-cosmology.dk, mm@roe.ac.uk, nrt3@leicester.ac.uk, \\ pja@hi.is, pmoller@eso.org, sts30@hi.is, tomedark-cosmology.dk
}

GRB-selected galaxies are broadly known to be faint, blue, young, star-forming dwarf galaxies. This insight, however, is based in part on heterogeneous samples of optically selected, lowerredshift galaxies. To study the statistical properties of GRB-selected galaxies we here introduce The Optically Unbiased GRB Host (TOUGH) complete sample of 69 X-ray selected Swift GRB host galaxies spanning the redshift range 0.03-6.30 and summarise the first results of a large observational survey of these galaxies.

Gamma-Ray Bursts 2012 Conference-GRB2012,

May 07-11, 2012

Munich, Germany

\footnotetext{
*Speaker.
} 


\section{Introduction}

Gamma-ray bursts (GRBs) arise from the deaths of short-lived stars [1]. Hence, their host galaxies bear important information about the nature of GRB progenitors and furthermore act as tracers of star formation at a very wide range of redshifts.

The host galaxy of the nearest known GRB host galaxy, that of GRB 980425/SN 1998bw at $z=0.0085$ [2], may be seen as representative of this population: it is a dwarf (SMC sized) starforming galaxy harbouring a highly star-forming region $[3,4]$. Indeed, in the BeppoSAX/HETE-II era, it was established that GRB host galaxies are typically sub-luminous, blue, young, high specific star-formation rate systems [5, 6, 7], with the GRBs originating from the UV/blue light of the galaxies $[8,9]$. The metallicities were found to be preferentially low $[10,11]$, providing an interesting constraint on progenitor models of GRBs, consistent, according to the mass-metallicity relation, with the finding that the stellar masses are generally low $[12,13]$. Early sub-mm (SCUBA) observations revealed a rare population of low-redshift $(z \sim 1)$ blue galaxies [14, 15], with somewhat elevated dust temperatures [16].

The above results are based on heterogeneous samples of mostly lower-redshift galaxies, e.g., relying on the existence of a bright optical afterglow for localisation and redshift determination.

At higher redshifts, GRB host galaxies are very faint, consistent with their being dwarf galaxies. For example, at $z=3.2$ (the redshift being determined via afterglow absorption spectroscopy) GRB 020124 [17, 18] and GRB 060526 [19] were found to be hosted by galaxies with $R>29.5$ and $R>28.5$, respectively. Remarkably, there are still no high-redshift $(z>5)$ GRB host galaxies robustly detected in emission [20], consistent with their being sub- $L^{*}$ galaxies.

However, more recent results have demonstrated that the host galaxies of dark GRBs are more chemically evolved and have higher masses [21], complicating the early simple picture of GRB host galaxies being sub-luminous, low-metallicity, highly star-forming blue galaxies (see also the contributions by Krühler, Levesque, and Perley to these proceedings).

\section{TOUGH}

Taking advantage of the launch of the Swift mission [22], The Optically Unbiased GRB Host (TOUGH) survey [23] was designed to define a large, carefully selected, homogeneous sample of 69 gamma-ray and X-ray selected galaxies, covering a wide redshift range. The selection criteria are detailed in [23]. Figure 1 shows the distribution of the peak gamma-ray flux of the TOUGH sample, suggesting a limit of about $0.3-0.4 \gamma \mathrm{cm}^{-2} \mathrm{~s}^{-1}$, almost an order of magnitude deeper than the sample of [24].

Our VLT spectroscopic follow-up campaign has led to 15 new redshifts [25, 26]. The redshift distributions before and after our observations are shown in Figure 2. The redshift distribution is discussed in more detail in [25] and in the contribution by Jakobsson et al. to these proceedings. As part of our spectroscopic observations we also specifically targeted 20 galaxies in the redshift range 1.8-4.5 to study their Ly $\alpha$ emission properties [27]. Seven of these have detections of Ly $\alpha$ emission and we can therefore exclude an early indication that Ly $\alpha$ emission is ubiquitous among GRB hosts. However, we confirm that $\operatorname{Ly} \alpha$ is stronger in GRB-selected galaxies than in fluxlimited samples of Lyman break galaxies. 


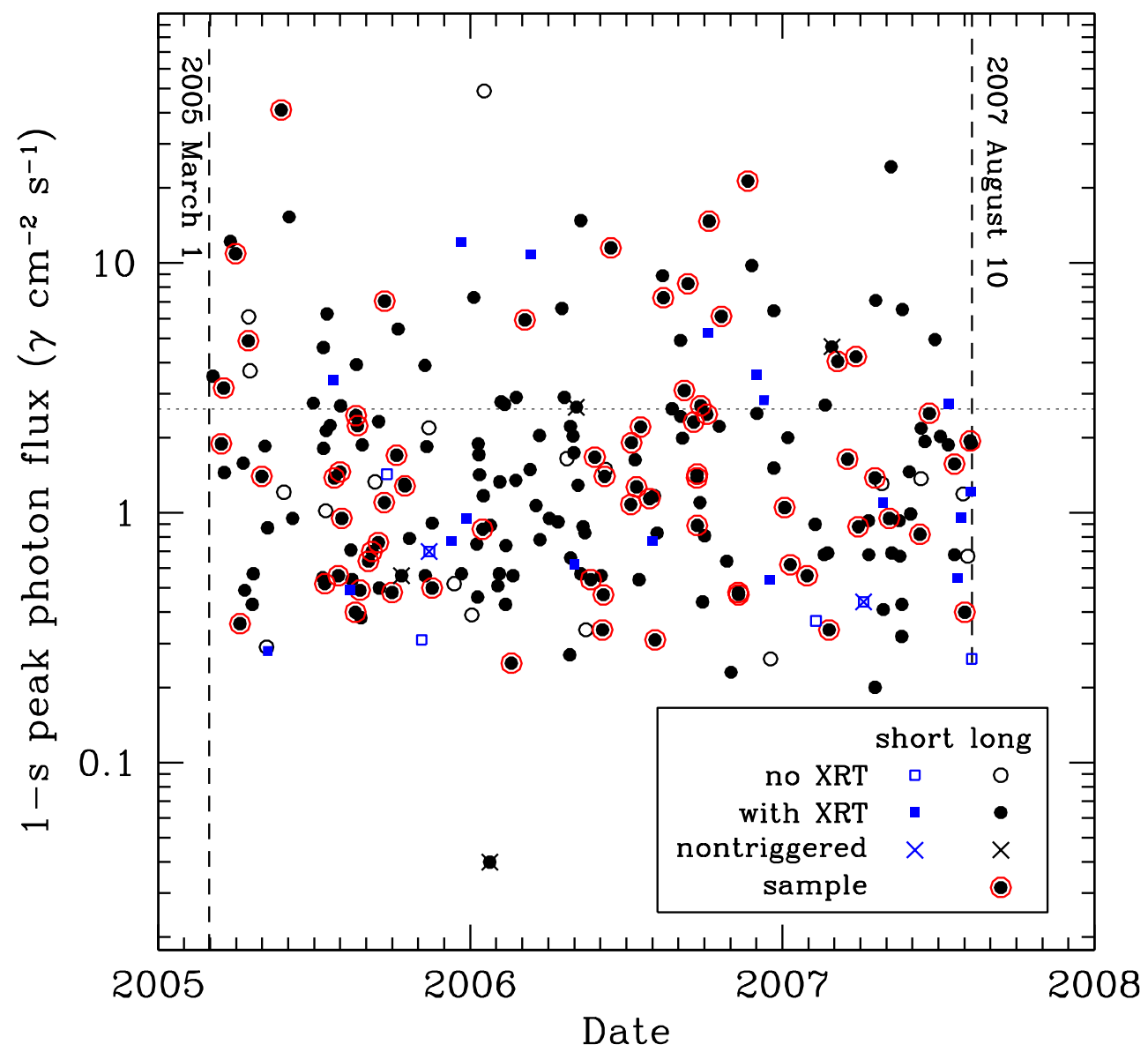

Figure 1: 1-s Swift/BAT peak flux (15-150 keV) for all Swift GRBs observed during the time span of the TOUGH survey (bracketed by vertical dashed lines). Empty circles: long bursts without an X-ray afterglow. Filled circles: long GRBs with Swift/XRT detection. Filled, encircled circles: GRBs obeying all TOUGH sample selection criteria. Squares: GRBs classified as short $\left(T_{90}<2 \mathrm{~s}\right)$. Crosses: nontriggered GRBs. There seems to be a constant and uniform detection level as a function of time. The horizontal line is the limiting peak flux limit adopted by [24]. From [23].

The survey also consists of deep $R$ - and $K$-band imaging. A key aspect of TOUGH is that we have imaged galaxies with no optical afterglows (i.e., localised in the X-rays by XRT to $2^{\prime \prime}$ precision) as well as those with optical afterglows. The resulting distribution of $R$-band magnitudes is shown in Figure 3. Remarkably, the host galaxies of GRBs with no optical afterglows are much brighter on average than those with an optical afterglow [23].

The colour distribution is shown in Figure 4. The median colours of XRT-only GRB host galaxies detected in the $K$-band are significantly redder, $R-K=3.7 \pm 0.3$, than those for which an optical afterglow was detected, $R-K=2.8 \pm 0.1$. GRBs without an optical afterglow may suffer stronger attenuation due to dust which may cause the optical afterglows to escape detection. 


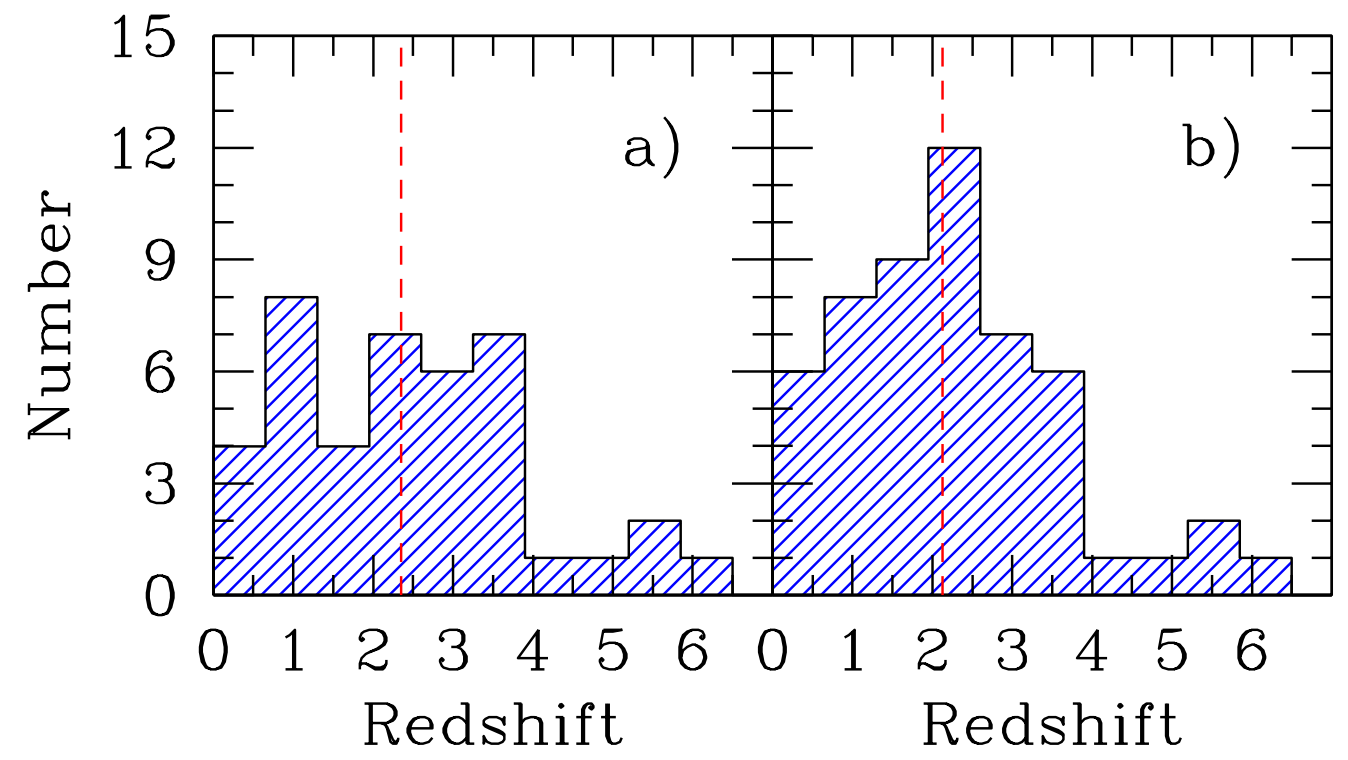

Figure 2: The redshift distribution of the TOUGH sample. In both panels the dashed vertical line indicates the median redshift. a) Before our survey, 38 redshifts were considered secure with a median redshift of $z=2.35$. b) Our TOUGH spectroscopic observations added 15 new redshifts and demonstrated that three redshifts reported in the literature are erroneous [25]. Here the median redshift is $z=2.14$. From [23].

In order to assess the star-formation rates (SFRs) of GRB hosts, we have also performed radio observations of TOUGH GRB hosts at $z<1$ [29]. We did not detect any TOUGH GRB hosts, which indicates that their average SFR is below $\sim 15 \mathrm{M}_{\odot} \mathrm{yr}^{-1}$. We also found that at least $65 \%$ of GRB hosts at $z<1$ have SFR $<100 \mathrm{M}_{\odot} \mathrm{yr}^{-1}$ and that at least $92 \%$ of them have $A_{V}<3$ mag. The distribution of SFRs and dust attenuation of GRB hosts at $z<1$ are consistent with those of other star-forming galaxies at similar redshifts (Figure 5).

Papers in preparation will address the luminosity function of GRB host galaxies, the X-ray absorption properties, metallicities of GRB host galaxies, and the correlation between prompt, afterglow, and host properties. Current and future work will concentrate on increasing the redshift completeness of the sample, extending the spectral coverage to longer wavelengths (radio, sub-mm, with Spitzer, Herschel, ALMA and EVLA) and imaging the fainter galaxies with HST. Enlarging the sample is also being considered. In parallel, detailed studies of TOUGH subsamples are in progress.

The TOUGH website is at: http: / / www . dark-cosmology.dk/TOUGH where catalogs and reduced data are made publicly available.

\section{Acknowledgments}

We thank the referee, Sandra Savaglio, for helpful comments. The Dark Cosmology Centre is funded by the Danish National Research Foundation. 


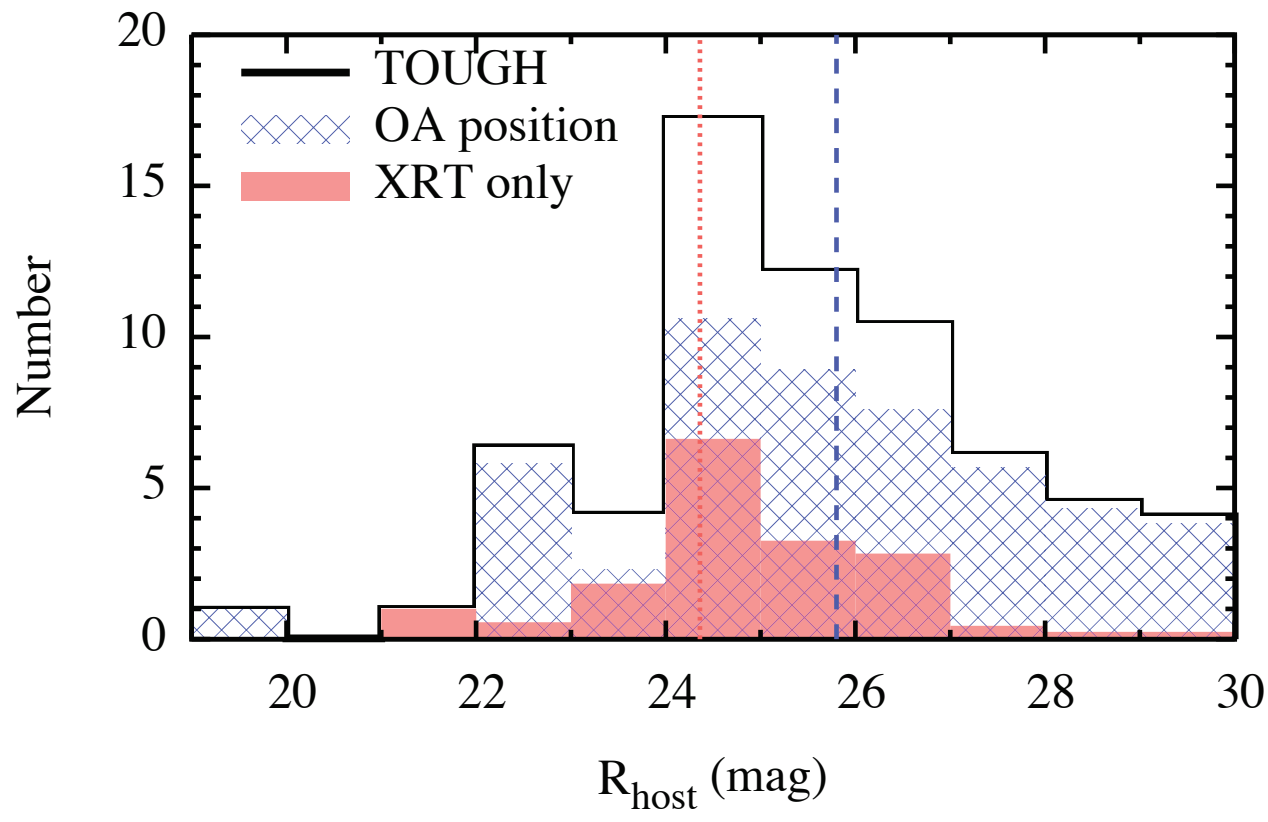

Figure 3: Distributions of host galaxy $R$-band magnitudes. Subsets of galaxies selected with a localisation from an optical afterglow and a localisation from Swift/XRT only are shown as hatched and solid histograms respectively. The corresponding median values are shown as dashed or dotted lines. Adapted from [23].

\section{References}

[1] J. Hjorth et al., A very energetic supernova associated with the $\gamma$-ray burst of 29 March 2003, Nature 423 (2003) 847 [astro-ph/0306347].

[2] T. J. Galama et al., An unusual supernova in the error box of the $\gamma$-ray burst of 25 April 1998, Nature 395 (1998) 670 [astro-ph/9806175].

[3] J. U. Fynbo et al., Hubble Space Telescope Space Telescope Imaging Spectrograph imaging of the host galaxy of GRB 980425/SN 1998bw, ApJ 542 (2000) L89 [astro-ph/ 0009014 ] .

[4] M. J. Michałowski, J. Hjorth, J. M. Castro Cerón \& D. Watson, The nature of GRB-selected submillimeter galaxies: hot and young, ApJ 672 (2008) 817 [arXiv:0708.3850].

[5] A. S. Fruchter et al., Hubble Space Telescope and Palomar imaging of GRB 990123: implications for the nature of gamma-ray bursts and their hosts, ApJ 519 (1999) L13 [astro-ph/9902236].

[6] E. Le Floc'h et al., Are the hosts of gamma-ray bursts sub-luminous and blue galaxies?, A\&A 400 (2003) 499 [astro-ph/0301149].

[7] L. Christensen, J. Hjorth \& J. Gorosabel, UV star-formation rates of GRB host galaxies, A\&A 425 (2004) 913 [astro-ph/ 0407066 ]. 


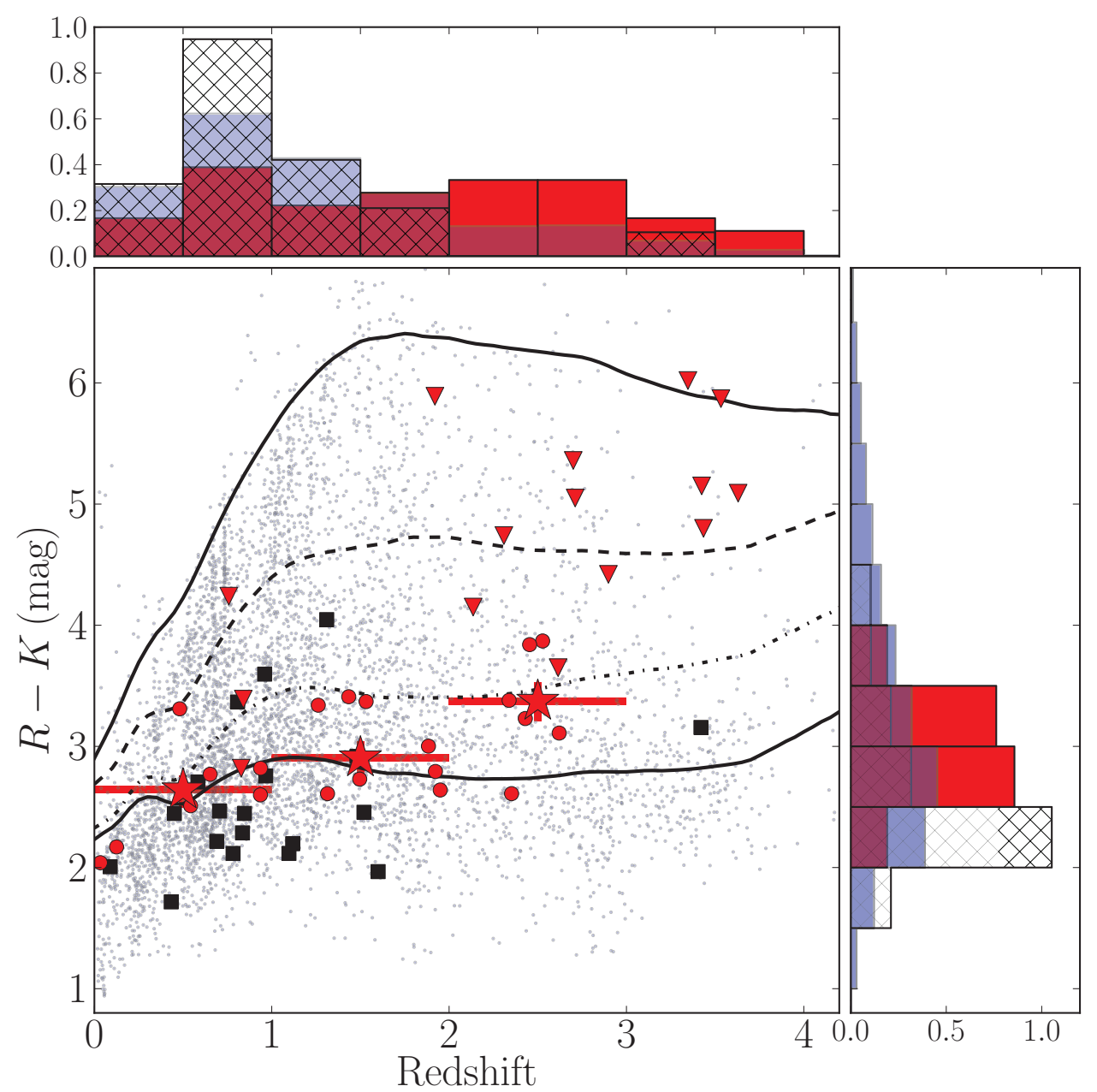

Figure 4: $R-K$ colour of TOUGH GRB hosts plotted against redshift. Red points represent TOUGH hosts detected in $R$ and $K$, while downward triangles represent those with $K$-band upper limits. Red stars illustrate the median $R-K$ colour over the indicated redshift interval. The data in black squares are the GRB-host sample of [13] and the grey background sample are field galaxies from the FIREWORKS catalog [28]. We also show redshift-dependent colours of different Hubble-type galaxies. These are (from red to blue $R-K$ colours): An old (3 Gyr) elliptical, S0 and Sbc spirals (1 Gyr) and a young (300 Myr) irregular galaxy at solar metallicity. These tracks have been derived from the PEGASE2 library integrated over the $R$ and $K$ bands.

[8] J. S. Bloom, S. R. Kulkarni \& S. G. Djorgovski, The observed offset distribution of gammaray bursts from their host galaxies: a robust clue to the nature of the progenitors, AJ $\mathbf{1 2 3}$ (2002) 1111 [astro-ph/0010176].

[9] A. S. Fruchter et al., Long $\gamma$-ray bursts and core-collapse supernovae have different environments, Nature 441 (2006) 463 [astro-ph/0603537].

[10] K. Z. Stanek et al., Protecting life in the Milky Way: metals keep the GRBs away, Acta Astron. 56 (2006) 333 [astro-ph / 0604113 ]. 


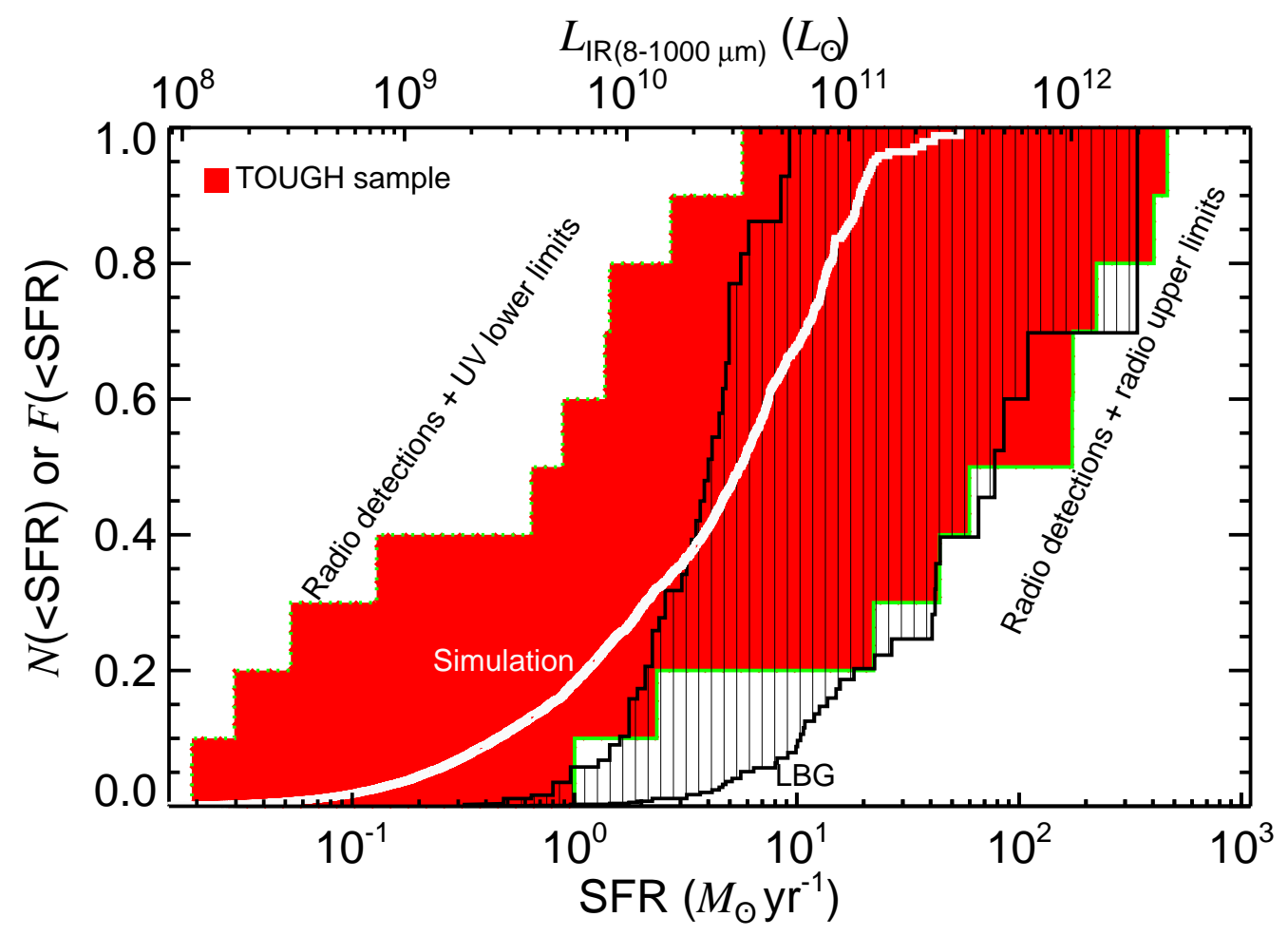

Figure 5: Cumulative distribution of SFRs of GRB hosts in the $z<1$ TOUGH sample (red area). The high-SFR boundaries (green solid lines) are constructed using the detections and limits of SFR $_{\text {radio, whereas }}$ the low-SFR boundaries (green dotted lines) are constructed using the SFR $\mathrm{UV}_{\text {for }}$ galaxies not detected in the radio. At least $63 \%(>15 / 24)$ of GRB hosts at $z \lesssim 1$ have $\mathrm{SFR}<100 \mathrm{M}_{\odot} \mathrm{yr}^{-1}$ and only $<8 \%$ $(<2 / 24)$ could have SFR $>500 \mathrm{M}_{\odot} \mathrm{yr}^{-1}$. For comparison, the SFR distributions of $z=0.51$ simulated galaxies (white curve) and $z \sim 1$ Lyman break galaxies (black curves; the right lines represent dust-corrected SFRs). These distributions were weighted by SFR (so they reflect the fraction of total star formation in the sample contributed by galaxies with SFRs lower than a given SFR) to allow a comparison with the GRB host population, which is likely selected based on SFRs. The current SFR limits imply that the GRB host population is consistent with star-forming galaxies at similar redshifts. Adapted from [29].

[11] M. Modjaz et al., Measured metallicities at the sites of nearby broad-lined Type Ic supernovae and implications for the supernovae gamma-ray burst connection, AJ 135 (2008) 1136 [astro-ph/0701246].

[12] J. M. Castro Cerón et al., On the distribution of stellar masses in gamma-ray burst host galaxies, ApJ 721 (2010) 1919 [arXiv:0803.2235].

[13] S. Savaglio, K. Glazebrook \& D. Le Borgne, The galaxy population hosting gamma-ray bursts, ApJ 691 (2009) 182 [arXiv:0803.2718].

[14] E. Berger et al., A submillimeter and radio survey of gamma-ray burst host galaxies: a glimpse into the future of star formation studies, ApJ 588 (2003) 99 [astro-ph/0210645]. 
[15] N. R. Tanvir et al., The submillimetre properties of gamma-ray burst host galaxies, MNRAS 352 (2004) 1073 [astro-ph/ 0406233 ].

[16] M. J. Michałowski et al., The properties of the host galaxy and the immediate environment of GRB 980425/SN 1998bw from the multiwavelength spectral energy distribution, ApJ 693 (2009) 347 [arXiv:0809.0508] .

[17] E. Berger et al., The faint optical afterglow and host galaxy of GRB 020124: implications for the nature of dark gamma-ray bursts, ApJ 581 (2002) 981 [astro-ph/0207320] .

[18] J. Hjorth et al., Very high column density and small reddening toward GRB 020124 at $z=3.20$, ApJ 597 (2003) 699 [astro-ph/0307331].

[19] C. C. Thöne et al., Photometry and spectroscopy of GRB 060526: a detailed study of the afterglow and host galaxy of a $z=3.2$ gamma-ray burst, A\&A 523 (2010) A70 [arXiv:0806.1182].

[20] N. R. Tanvir et al., Star formation in the early universe: beyond the tip of the iceberg, ApJ 754 (2012) 46 [arXiv:1201.6074].

[21] T. Krühler et al., The SEDs and host galaxies of the dustiest GRB afterglows, A\&A 534 (2011) A108 [arXiv:1108.0674].

[22] N. Gehrels et al., The Swift gamma-ray burst mission, ApJ 611 (2004) 1005 [astro-ph/0405233].

[23] J. Hjorth et al., The optically unbiased GRB host (TOUGH) survey. I. Survey design and catalogs, ApJ 756 (2012) 187 [arXiv:1205.3162].

[24] R. Salvaterra et al., A complete sample of bright Swift long gamma-ray bursts. I. Sample presentation, luminosity function and evolution, ApJ 749 (2012) 68 [arXiv:1112.1700] .

[25] P. Jakobsson et al., The optically unbiased GRB host (TOUGH) survey. III. Redshift distribution, ApJ 752 (2012) 62 [arXiv: 1205.3490].

[26] T. Krühler et al., The optically unbiased GRB host (TOUGH) survey. V. VLT/Xshooter emission-line redshifts for Swift GRBs at $z \sim 2$, ApJ, in press (2012) [arXiv:1205.4036].

[27] B. Milvang-Jensen et al., The optically unbiased GRB host (TOUGH) survey. IV. Lyman- $\alpha$ emitters, ApJ 756 (2012) 25 [arXiv:1205.3779].

[28] S. Wuyts et al., FIREWORKS U38-to-24 $\mu$ m photometry of the GOODS Chandra Deep FieldSouth: multiwavelength catalog and total infrared properties of distant $K_{S^{-}}$-selected galaxies, ApJ 682 (2008) 985 [arXiv: 0804 .0615].

[29] M. J. Michałowski et al., The optically unbiased GRB host (TOUGH) survey. VI. Radio observations at $z<1$ and consistency with typical star-forming galaxies, ApJ 755 (2012) 85 [arXiv:1205.4239]. 